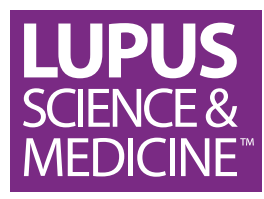

\title{
Discoid lupus erythematosus skin lesion distribution and characteristics in Black patients: a retrospective cohort study
}

\author{
Adrienne K Joseph, ${ }^{1}$ Brandon Windsor, ${ }^{2}$ Linda S Hynan, ${ }^{3}$ Benjamin F Chong (i) ${ }^{1}$
}

To cite: Joseph AK, Windsor B, Hynan LS, et al. Discoid lupus erythematosus skin lesion distribution and characteristics in Black patients: a retrospective cohort study. Lupus Science \& Medicine 2021;8:e00514. doi:10.1136/ lupus-2021-000514

\section{- Additional supplemental} material is published online only. To view, please visit the journal online (http://dx.doi.org/10. 1136/lupus-2021-000514).

Received 27 April 2021 Accepted 29 October 2021

D) Check for updates

(C) Author(s) (or their employer(s)) 2021. Re-use permitted under CC BY-NC. No commercial re-use. See rights and permissions. Published by BMJ.

${ }^{1}$ Dermatology, The University of Texas Southwestern Medical Center, Dallas, Texas, USA

${ }^{2}$ Population and Data Sciences, The University of Texas Southwestern Medical Center, Dallas, Texas, USA

${ }^{3}$ Population and Data Sciences, Psychiatry, The University of Texas Southwestern Medical Center, Dallas, Texas, USA

Correspondence to Dr Benjamin F Chong; Ben. Chong@UTSouthwestern.edu

\section{ABSTRACT}

Objective Epidemiological studies have shown that discoid lupus erythematosus (DLE) has a higher incidence and prevalence in racial/ethnic minority groups, particularly Black individuals. The objective of this retrospective cohort study was to identify the differences in DLE lesion distribution and characteristics in Black individuals compared with non-Black individuals. Methods 183 patients with DLE (112 Black patients and 71 non-Black patients) with a reported race/ ethnicity and Cutaneous Lupus Erythematosus Disease Area and Severity Index (CLASI) scores were included in this retrospective cohort study. Univariate analysis was performed to determine significant differences in demographic data, clinical characteristics, DLE lesion distribution and DLE lesion characteristics in Black and non-Black patients with DLE. Multivariable logistic regression was preformed to determine significant predictors of DLE lesion location and characteristics. Results Black patients with DLE had worse baseline CLASI damage scores compared with non-Black patients with DLE (median (IQR): 10.0 (6.0-14.5) vs 6.0 (3.0-10.0), $\mathrm{p}<0.001$ ) and had 48.9 greater odds of dyspigmentation in any anatomical location $(p<0.001)$. Black patients had 2.54 greater odds of having scalp involvement $(p=0.015)$ and 1.97 greater odds of having ear involvement $(p=0.032)$ compared with non-Black patients. Black patients also had greater odds of scalp dyspigmentation $(0 R=5.85, p<0.001)$, ear dyspigmentation $(0 R=2.89$, $\mathrm{p}=0.001)$ and scarring alopecia $(0 \mathrm{R}=3.00, \mathrm{p}=0.001)$ compared with non-Black patients.

Conclusions Signs of disease damage, particularly ear dyspigmentation, scalp dyspigmentation and scarring alopecia, can more frequently affect Black patients with DLE. Recognising differences in clinical presentation of DLE among Black patients can assist future efforts with understanding biological, cultural, psychosocial and systemic factors that influence DLE presentation and outcomes in Black patients and may guide clinicians when counselling Black patients.

\section{INTRODUCTION}

Discoid lupus erythematosus (DLE) is a form of chronic cutaneous lupus erythematosus (CLE) that starts with erythematous scaly

\section{Key messages}

What is already known about this subject?

- Epidemiological studies have shown that discoid lupus erythematosus (DLE) has a higher incidence and prevalence in Black individuals.

- Black patients with DLE present with greater disease damage earlier in their disease course.

What does this study add?

- We have identified important differences in DLE lesion location and lesion characteristics in Black patients with DLE compared with non-Black patients with DLE.

How might this impact on clinical practice or future developments?

- Our findings will help clinicians recognise ear dyspigmentation, scalp dyspigmentation and scarring alopecia as prominent features of DLE in Black patients and counsel Black patients on treating signs and symptoms of DLE.

- Recognising differences in clinical presentation of DLE in Black patients can assist future efforts with understanding biological, cultural, psychosocial and systemic factors that influence DLE presentation and outcomes in Black patients.

plaques and resolves with dyspigmentation and scarring. Common sites for DLE lesions include scalp, ears and cheeks. Epidemiological studies suggest that DLE has a higher incidence and prevalence in Black individuals. Black patients may also present with greater disease damage earlier in their disease course. However, racial differences in clinical presentation among patients with DLE are not well understood.

Understanding racial differences in skin disease presentations has important implications for addressing disparities. In psoriasis, skin lesions in racial and ethnic minority groups present with greater severity and resolve with more dyspigmentation. ${ }^{1}$ Additionally, Black and Asian patients may present 
with more scalp psoriasis. Recognition of these racial and ethnic differences in psoriasis has given better insight into the biopsychosocial factors that influence disease presentation and outcomes. ${ }^{1}$

Recognising racial differences in dermatological disease and understanding the elements that drive differences may also be of importance to patients. One group of Black patients with dermatological disease reported higher satisfaction with clinicians who were perceived to have more knowledge on Black patients' skin and hair. ${ }^{2}$ Patients specifically described the importance of clinician understanding of differences in cultural, environmental and social factors affecting Black patients' skin and hair.

We sought to identify how DLE differentially presents in Black patients to give clinicians better understanding into what aspects of DLE may disproportionately affect Black patients and to help clinicians better provide recommendations to Black patients with DLE.

\section{METHODS}

\section{Patient population}

This was a retrospective cohort study of patients with DLE recruited from the University of Texas Southwestern (UTSW) CLE Registry from January 2009 to June 2020. Patients were seen in outpatient dermatology clinics at UTSW Medical Center and Parkland Health and Hospital System in Dallas, Texas. All DLE diagnoses were confirmed by a dermatologist (BFC) via clinicopathological correlation. Adult patients with DLE with a reported race/ethnicity and Cutaneous Lupus Erythematosus Disease Area and Severity Index (CLASI) scores were included in this study. Patients were excluded if they had an additional CLE subtype or if they were $<18$ years old. All participants gave informed consent.

\section{Variables}

DLE lesion locations and characteristics based on CLASI scores were the primary outcome variables. The CLASI is a validated instrument that measures severity of disease activity and disease damage. ${ }^{3}$ Disease activity characteristics include erythema, scale/hyperkeratosis, mucous membrane involvement and non-scarring hair loss. Disease damage components include dyspigmentation, scarring and scarring hair loss. The primary predictor variable was race, which was self-reported and categorised into Black patients and non-Black patients. Additional predictor variables included age at initial visit, gender, disease duration at initial visit, follow-up duration, smoking status and presence or absence of SLE.

\section{Statistical analysis}

Categorical variables were reported with frequencies and percentages. Continuous variables were reported with median and interquartile range (IQR). Univariate analyses (ie, Mann-Whitney $U$ test for continuous variables

Table 1 Demographics and clinical characteristics of 112 Black and 71 non-Black patients with DLE

\begin{tabular}{|c|c|c|c|}
\hline Demographics and clinical characteristics & $\begin{array}{l}\text { Black patients } \\
(n=112)\end{array}$ & $\begin{array}{l}\text { Non-Black patients* } \\
(n=71)\end{array}$ & $P$ value \\
\hline Age at initial visit (years), median (IQR) & $50.0(39.2-56.9)$ & $46.0(35.5-54.3)$ & $0.041 \dagger$ \\
\hline Age at diagnosis (years), median (IQR) & $40.0(30.0-49.0)$ & $36.0(27.0-47.0)$ & $0.266 \dagger$ \\
\hline Gender, n (\%) & & & $0.716 \ddagger$ \\
\hline Male & $18(16)$ & $10(14)$ & \\
\hline Female & $94(84)$ & $61(86)$ & \\
\hline Disease duration at initial visit (years), median (IQR) & $4.4(0.9-12.4)$ & $3.5(1.0-10.2)$ & $0.964 \dagger$ \\
\hline Follow-up duration (years), median (IQR) & $0.4(0.0-2.9)$ & $0.4(0.0-2.9)$ & $0.967 \dagger$ \\
\hline SLE diagnosis, $\mathrm{n}(\%)$ & & & $0.222 \ddagger$ \\
\hline Yes & $48(43)$ & $24(34)$ & \\
\hline No & $64(57)$ & $47(66)$ & \\
\hline Smoking status, $\mathrm{n}(\%) \S$ & & & $0.451 \ddagger$ \\
\hline Current smoker & $42(38)$ & $25(39)$ & \\
\hline Past smoker & $25(23)$ & $10(16)$ & \\
\hline Never smoked & $43(39)$ & $30(46)$ & \\
\hline Baseline CLASI activity score, median (IQR) & $4.5(2.0-9.0)$ & $5.0(2.0-10.0)$ & $0.912 \dagger$ \\
\hline Baseline CLASI damage score, median (IQR) & $10.0(6.0-14.5)$ & $6.0(3.0-10.0)$ & $<0.001 \dagger$ \\
\hline
\end{tabular}

*Non-Black patients consisted of 45 non-Hispanic White patients, 18 Hispanic patients, 5 Asian patients, 2 mixed race patients and 1 Middle Eastern patient with DLE.

†P value was calculated with Mann-Whitney $U$ test.

$\ddagger P$ value was calculated with $\chi^{2}$ test.

$\S$ Smoking status was missing for 8 patients.

CLASI, Cutaneous Lupus Erythematosus Disease Activity and Severity Index; DLE, discoid lupus erythematosus. 
Table 2 DLE lesion distribution based on CLASI scores in 112 Black and 71 non-Black patients

\begin{tabular}{|c|c|c|c|c|c|c|}
\hline $\begin{array}{l}\text { Anatomical } \\
\text { location }\end{array}$ & $\begin{array}{l}\text { Black patients } \\
\text { n (\%) }\end{array}$ & $\begin{array}{l}\text { Non-Black patients } \\
\text { n (\%) }\end{array}$ & $\begin{array}{l}\text { Univariate } \\
\text { OR }(95 \% \mathrm{CI})\end{array}$ & P value* & $\begin{array}{l}\text { Multivariable } \\
\text { OR }(95 \% \mathrm{Cl}) \dagger \ddagger\end{array}$ & $\mathrm{P}$ value † \\
\hline Scalp & 97 (87) & $51(72)$ & 2.54 (1.12 to 5.79$)$ & 0.013 & 2.54 (1.20 to 5.37$)$ & 0.015 \\
\hline Nose/malar area & $60(59)$ & $40(56)$ & 0.89 (0.47 to 1.70$)$ & 0.714 & - & - \\
\hline Rest of face & $63(56)$ & $37(53)$ & 1.18 (0.62 to 2.24$)$ & 0.584 & - & - \\
\hline $\begin{array}{l}\text { Posterior neck/ } \\
\text { shoulders }\end{array}$ & $22(20)$ & $20(28)$ & 0.62 (0.29 to 1.33$)$ & 0.181 & - & - \\
\hline Chest & 20 (18) & $18(25)$ & 0.64 (0.29 to 1.41$)$ & 0.223 & - & - \\
\hline Abdomen & $10(9)$ & $1(1)$ & N/Aף & $0.053^{\star *}$ & - & - \\
\hline Legs & $23(21)$ & $18(25)$ & 0.76 (0.36 to 1.65$)$ & 0.446 & - & - \\
\hline Feet & $20(18)$ & $8(11)$ & 1.71 (0.67 to 4.77 ) & 0.228 & - & - \\
\hline $\begin{array}{l}\text { Mucous } \\
\text { membrane }\end{array}$ & $15(13)$ & $9(13)$ & 1.07 (0.41 to 2.94$)$ & 0.889 & - & - \\
\hline
\end{tabular}

${ }^{*} P$ value was calculated with $\chi^{2}$ test unless otherwise specified.

$\dagger^{\prime}-$ ' indicates that race was not a significant factor in multivariable analyses.

$\ddagger$ Predictor variables (race, age at initial visit, gender, disease duration at initial visit, follow-up duration, smoking status, presence of SLE) were included in multivariable analyses.

§Race was the only significant predictor variable for scalp involvement. Race and age at initial visit $(\mathrm{OR}=0.97,95 \% \mathrm{Cl} 0.95$ to $0.99, \mathrm{p}=0.015)$ were significant predictor variables for ear involvement.

IOR not calculated due to a low number of patients with abdomen involvement in the non-black patient group.

${ }^{* *} \mathrm{P}$ value was calculated with Fisher's exact test.

CI, confidence interval; CLASI, Cutaneous Lupus Erythematosus Disease Area and Severity Index; DLE, discoid lupus erythematous; N/A, not available; OR, odds ratio.

and $\chi^{2}$ or Fisher's exact test, as appropriate, for categorical variables) were performed to determine demographic differences and to compare lesion location and lesion characteristics between Black and non-Black patients. We selected a $p$ value cut-off of 0.01 for univariate analyses, as other methods of accounting for multiple comparisons were considered too conservative. Multivariable logistic regression was performed to identify predictor variables, chosen a priori (race, age at initial visit, gender, disease duration at initial visit, follow-up duration, smoking status, presence of SLE), associated with DLE lesion location and lesion characteristics. Since this is a pilot study, $\mathrm{p}<0.05$ was considered statistically significant for multivariable logistic regression analyses. Univariate analysis was performed with STATA V.16.1. Multivariable logistic regression was performed with SPSS V.26.

\section{RESULTS}

Demographic and clinical characteristics of Black and nonBlack patients with DLE

A total of 183 patients with DLE, 112 Black patients and 71 non-Black patients, were included in this study. Non-Black patients included 45 non-Hispanic White patients, 18 Hispanic patients, 5 Asian patients, 2 mixed race patients and 1 Middle Eastern patient with DLE. Black patients with DLE (median=10.0, IQR 6.0-14.5) had higher baseline CLASI damage scores compared with non-Black patients (median=6.0, IQR 3.0-10.0) $(p<0.001)$. No differences were observed between Black and non-Black individuals in other demographic characteristics (table 1).

\section{DLE lesion distribution in Black and non-Black patients}

Univariate analyses showed no significant differences in anatomical location of DLE lesions between Black and non-Black patients (table 2). However, multivariable logistic regression revealed that Black patients with DLE had greater odds of having scalp (odds ratio $(\mathrm{OR})=2.54$, $95 \%$ confidence interval (CI) 1.20 to $5.37, \mathrm{p}=0.015$ ) and ear $(\mathrm{OR}=1.97,95 \%$ CI 1.06 to $3.68, \mathrm{p}=0.032)$ involvement compared with non-Black patients with DLE.

\section{DLE lesion characteristics in Black and non-Black patients}

Black patients were more affected by the presence of dyspigmentation in any location (99\% vs $79 \%, \mathrm{p}<0.001)$. Black patients with DLE were more likely to have dyspigmentation of the scalp ( $82 \%$ vs $48 \%, \mathrm{p}<0.001)$ and ears $(56 \%$ vs $35 \%, \mathrm{p}=0.006)$ compared with non-Black patients. Black patients with DLE also more frequently 


\begin{tabular}{|c|c|c|c|c|c|}
\hline $\begin{array}{l}\text { Anatomical location and } \\
\text { lesion characteristics }\end{array}$ & $\begin{array}{l}\text { Black patients } \\
\mathrm{n}(\%)\end{array}$ & $\begin{array}{l}\text { Non-Black patients } \\
\mathrm{n}(\%)\end{array}$ & $P$ value* & $\begin{array}{l}\text { Multivariable } \\
\text { OR }(95 \% \mathrm{Cl}) \dagger \ddagger \S\end{array}$ & $P$ value $† £ \S$ \\
\hline Erythema in any location & $99(88)$ & $63(89)$ & 0.944 & N/A & $\mathrm{N} / \mathrm{A}$ \\
\hline Scale in any location & $78(69)$ & $53(75)$ & 0.464 & N/A & $\mathrm{N} / \mathrm{A}$ \\
\hline $\begin{array}{l}\text { Dyspigmentation in any } \\
\text { location }\end{array}$ & $111(99)$ & $56(79)$ & $<0.001$ & 48.94 (5.82 to 411.77$)$ १ & $<0.001$ \\
\hline Scarring in any location & $100(89)$ & $55(77)$ & 0.030 & N/A & N/A \\
\hline \multicolumn{6}{|l|}{ Scalp } \\
\hline Erythema & $75(59)$ & $42(67)$ & 0.284 & N/A & $\mathrm{N} / \mathrm{A}$ \\
\hline Scale/hypertrophy & $58(52)$ & $34(48)$ & 0.607 & N/A & N/A \\
\hline Dyspigmentation & $92(82)$ & $32(48)$ & $<0.001$ & $5.85(2.94 \text { to } 11.61)^{\star *}$ & $<0.001$ \\
\hline Scarring alopecia & $89(79)$ & $40(56)$ & 0.001 & 3.00 (1.56 to 5.78$) \dagger \dagger$ & 0.001 \\
\hline Non-scarring alopecia & $49(44)$ & $29(41)$ & 0.699 & N/A & N/A \\
\hline \multicolumn{6}{|l|}{ Ears } \\
\hline Erythema & $36(32)$ & $20(28)$ & 0.570 & N/A & N/A \\
\hline Scale/hypertrophy & $34(30)$ & $22(31)$ & 0.928 & N/A & N/A \\
\hline Dyspigmentation & $63(56)$ & $25(35)$ & 0.006 & 2.89 (1.50 to 5.54$) \neq \ddagger$ & 0.001 \\
\hline Scarring & $31(28)$ & $14(20)$ & 0.223 & N/A & $\mathrm{N} / \mathrm{A}$ \\
\hline
\end{tabular}

${ }^{*} \mathrm{P}$ value was calculated with $\chi^{2}$ test.

†Blank values represented by '-' indicate variables for which race was not a significant factor in the multivariable analyses.

$\ddagger N / A$ represents values for which multivariable analyses were not performed.

§Predictor variables (race, age at initial visit, gender, disease duration at initial visit, follow-up duration, smoking status, presence of SLE) were included in multivariable analyses.

IRace and age at initial visit $(\mathrm{OR}=0.92,95 \% \mathrm{Cl} 0.88$ to $0.97, \mathrm{p}=0.002)$ were significant predictors of dyspigmentation in any anatomical location.

${ }^{\star \star}$ Race and follow-up duration $(\mathrm{OR}=1.17,95 \% \mathrm{Cl} 1.01$ to $1.36, \mathrm{p}=0.04)$ were significant predictors of scalp dyspigmentation.

$\dagger+$ Race was the only significant predictor of scarring alopecia.

$\ddagger \ddagger R$ ace and age at initial visit $(\mathrm{OR}=0.96,95 \% \mathrm{Cl} 0.94$ to $0.98, \mathrm{p}=0.001)$ were also significant predictors of ear dyspigmentation.

$\mathrm{Cl}$, confidence interval; CLASI, Cutaneous Lupus Erythematosus Disease Area and Severity Index; DLE, discoid lupus erythematous; N/A, not available; OR, odds ratio.

had scarring alopecia $(79 \%$ vs $56 \%, \mathrm{p}=0.001)$ (table 3$)$. In multivariable analyses, Black patients had greater odds of having dyspigmentation in any anatomical location (48.94 (5.82-411.77), $\mathrm{p}<0.001)$, ear dyspigmentation (2.89 (1.50-5.54), $\mathrm{p}=0.001)$, scalp dyspigmentation (5.85 $(2.94-11.61), \mathrm{p}<0.001)$ and scalp scarring (3.00 (1.56$5.78), \mathrm{p}=0.001$ ) compared with non-Black patients. There were non-significant racial differences in DLE lesion characteristics in other body areas (online supplemental table 1).

\section{DISCUSSION}

Dyspigmentation was a prominent feature of DLE in Black patients. Although our study looked at the presence or absence of dyspigmentation, not severity, due to the nature of CLASI scoring, dyspigmentation may be more obvious in patients with darker skin types. Skin colour is one aspect associated with race, and patients with darker skin may experience more frequent and more severe dyspigmentation regardless of racial background. Greater contrast with surrounding unaffected skin may make hypopigmentation more noticeable, while hyperpigmentation may be more prominent due to increased melanin production in those with darker skin types. ${ }^{4}$ Clinicians can discuss pigmentary concerns with patients and provide them with options to improve the appearance of dyspigmentation, such as cosmetic camouflage, whenever it is bothersome.

Black patients also had increased odds of scalp and ear involvement and were more frequently affected by ear dyspigmentation, scalp dyspigmentation and scarring alopecia. It is possible that Black patients with DLE develop skin damage of the scalp and ear more quickly than non-Black patients, ${ }^{5}$ given that both patient groups reported similar disease activity and follow-up duration. It is unclear what factors promote increased scalp involvement and damage among black patients with DLE, but we postulate that it is likely multifactorial. Cultural haircare practices among black patients may contribute to more frequent and more severe scarring alopecia, especially since DLE lesions may koebnerise. ${ }^{6}$ Traction alopecia and central centrifugal cicatricial alopecia are other forms of alopecia that disproportionately affect Black patients. In traction alopecia, cultural haircare practices have been implicated in its pathogenesis, while in central centrifugal cicatricial alopecia both cultural haircare 
practices and genetics have been implicated. ${ }^{7-9}$ Contributing factors to scalp and ear involvement and damage in DLE warrant further investigation.

Black patients with DLE, or those suspected of having DLE, can be examined for scalp and ear involvement to assist in initiation of treatment of these lesions. Prior studies have found that some Black patients perceive that some clinicians lack understanding of and have discomfort with Black individuals' skin and hair. ${ }^{2} 10$ This may serve as a barrier to Black patients with DLE seeking and receiving treatment. A culturally competent approach to examination and treatment can be used by clinicians to provide better care to patients. Recommendations have previously been outlined for examining the hair and scalp of patients with tightly coiled hair. ${ }^{11}$ Black patients with DLE can be counselled on gentle haircare practices to reduce further scalp damage. ${ }^{7}$ Providers can educate themselves on common haircare practices among Black individuals to further refine their recommendations. ${ }^{7}$

Limitations of this study include its single-centre design and small sample size. However, the racial diversity of the UTSW CLE cohort is consistent with other epidemiological studies of DLE. ${ }^{12} 13$ Further multicentre studies are needed to confirm our findings. Future efforts could further explore how specific factors, including cultural hair/skin care practices, physician-patient relationships, and societal and systemic influences, contribute to racial differences observed among Black patients with DLE. Additionally, our study focused only on Black patients with DLE. Future studies are needed to examine how DLE differentially presents in other racial and ethnic groups to have a better understanding of what factors uniquely impact those populations.

Our findings will help clinicians understand racial differences in DLE presentation, which may lead to increased consideration of intrinsic and extrinsic influences on treatment needs and preferences of Black patients. This study can also assist future efforts with understanding which factors influence DLE presentation and outcomes.

Acknowledgements The authors would like to thank Rebecca Vasquez, Andrew Kim, Daniel Grabell, Noelle Teske, Tina Vinoya, Jack O'Brien, Elaine Kunzler, Stephanie Florez-Pollack, Jennifer Coias, Smriti Prasad, Danielle Lin, Jenny Raman, Justin Raman, Jane Zhu, Samantha Black and Laila Abbas for recruiting patients. The authors would like to thank the participants of the University of Texas Southwestern CLE Registry for their contributions to lupus research.

Contributors All authors contributed to the study conception and design. Data collection and analysis were performed by AKJ, BW and LH. The first draft of the manuscript was written by AKJ, and all authors commented on the subsequent versions of the manuscript. All authors read and approved the final manuscript.

Funding The research reported in this publication was supported by the National Institute of Arthritis and Musculoskeletal and Skin Diseases of the National Institutes of Health under award number K23AR0614415.
Competing interests BFC is an investigator for Daavlin, Biogen, Pfizer and Amgen. He is a consultant for Viela Bio, Beacon Bioscience, Bristol Myers Squibb, EMD Serono and Principia Biopharma.

Patient consent for publication Not required.

Ethics approval The study was approved by the UTSW Institutional Review Board. Provenance and peer review Not commissioned; externally peer reviewed.

Supplemental material This content has been supplied by the author(s). It has not been vetted by BMJ Publishing Group Limited (BMJ) and may not have been peer-reviewed. Any opinions or recommendations discussed are solely those of the author(s) and are not endorsed by BMJ. BMJ disclaims all liability and responsibility arising from any reliance placed on the content. Where the content includes any translated material, BMJ does not warrant the accuracy and reliability of the translations (including but not limited to local regulations, clinical guidelines, terminology, drug names and drug dosages), and is not responsible for any error and/or omissions arising from translation and adaptation or otherwise.

Open access This is an open access article distributed in accordance with the Creative Commons Attribution Non Commercial (CC BY-NC 4.0) license, which permits others to distribute, remix, adapt, build upon this work non-commercially, and license their derivative works on different terms, provided the original work is properly cited, appropriate credit is given, any changes made indicated, and the use is non-commercial. See: http://creativecommons.org/licenses/by-nc/4.0/.

ORCID iD

Benjamin F Chong http://orcid.org/0000-0002-4092-7658

\section{REFERENCES}

1 Nicholas MN, Chan AR, Hessami-Booshehri M. Psoriasis in patients of color: differences in morphology, clinical presentation, and treatment. Cutis 2020;106:E10:7-10.

2 Gorbatenko-Roth K, Prose N, Kundu RV, et al. Assessment of black patients' perception of their dermatology care. JAMA Dermatol 2019;155:1129.

3 Albrecht J, Taylor L, Berlin JA, et al. The CLASI (cutaneous lupus erythematosus disease area and severity index): an outcome instrument for cutaneous lupus erythematosus. $J$ Invest Dermatol 2005;125:889-94.

4 Callender VD, St Surin-Lord S, Davis EC, et al. Postinflammatory hyperpigmentation: etiologic and therapeutic considerations. Am J Clin Dermatol 2011;12:87-99.

5 Verma SM, Okawa J, Propert KJ, et al. The impact of skin damage due to cutaneous lupus on quality of life. $\mathrm{Br} J$ Dermatol 2014;170:315-21.

6 Concha JSS, Werth VP. Alopecias in lupus erythematosus. Lupus Sci Med 2018;5:e000291.

7 Haskin A, Aguh C. All hairstyles are not created equal: what the dermatologist needs to know about black hairstyling practices and the risk of traction alopecia (TA). J Am Acad Dermatol 2016;75:606-11.

8 Malki L, Sarig O, Romano M-T, et al. Variant PADI3 in Central Centrifugal Cicatricial Alopecia. N Engl J Med 2019;380:833-41.

9 BS L, Maibach HI. Ethnic skin and hair and other cultural considerations. Springer International Publishing, 2021.

10 Gathers RC, Mahan MG. African American women, hair care, and health barriers. J Clin Aesthet Dermatol 2014;7:26-9.

11 Grayson C, Heath C. An approach to examining tightly coiled hair among patients with hair loss in Race-Discordant patient-physician interactions. JAMA Dermatol 2021;157:505-6.

12 Drenkard C, Parker S, Aspey LD, et al. Racial disparities in the incidence of primary chronic cutaneous lupus erythematosus in the southeastern us: the Georgia lupus registry. Arthritis Care Res 2019;71:95-103.

13 Izmirly P, Buyon J, Belmont HM, et al. Population-Based prevalence and incidence estimates of primary discoid lupus erythematosus from the Manhattan lupus surveillance program. Lupus Sci Med 2019;6:e000344. 